\title{
A study of muon pair production and evidence for tau pair production in photon-photon collisions at LEP
}

\author{
OPAL Collaboration
}

R. Akers ${ }^{16}$, G. Alexander ${ }^{23}$, J. Allison ${ }^{16}$, K.J. Anderson ${ }^{9}$, S. Arcelli' ${ }^{2}$ A. Astbury ${ }^{28}$, D. Axen ${ }^{29}$, G. Azuelos ${ }^{18, a}$, J.T.M. Baines ${ }^{16}$, A.H. Ball ${ }^{17}$, J. Banks ${ }^{16}$, R.J. Barlow ${ }^{16}$, S. Barnett ${ }^{16}$, R. Bartoldus ${ }^{3}$, J.R. Batley ${ }^{5}$, G. Beaudoin ${ }^{18}$, A. Beck ${ }^{23}$, G.A. Beck ${ }^{13}$, J. Becker ${ }^{10}$, C. Beeston ${ }^{16}$, T. Behnke ${ }^{27}$, K.W. Bell ${ }^{20}$, G. Bella ${ }^{23}$, P. Bentkowski ${ }^{18}$, P. Berlich ${ }^{10}$, S. Bethke ${ }^{11}$, O. Biebel ${ }^{3}$, I.J. Bloodworth ${ }^{1}$, P. Bock ${ }^{11}$, B. Boden ${ }^{3}$, H.M. Bosch ${ }^{11}$, M. Boutemeur ${ }^{18}$, H. Breuker ${ }^{8, b}$, P. Bright-Thomas ${ }^{25}$, R.M. Brown ${ }^{20}$, A. Buijs ${ }^{8}$, H.J. Burckhart ${ }^{8}$, C. Burgard ${ }^{27}$, P. Capiluppi ${ }^{2}$, R.K. Carnegie ${ }^{6}$, A.A. Carter ${ }^{13}$, J.R. Carter ${ }^{5}$, C.Y. Chang ${ }^{17}$, D.G. Charlton ${ }^{8}$, S.L. Chu ${ }^{4}$, P.E.L. Clarke ${ }^{15}$, J.C. Clayton ${ }^{1}$ I. Cohen ${ }^{23}$, J.E. Conboy ${ }^{15}$, M. Cooper ${ }^{22}$, M. Coupland ${ }^{14}$, M. Cuffiani' ${ }^{2}$, S. Dado ${ }^{22}$, G.M. Dallavalle ${ }^{2}$, S. De Jong ${ }^{13}$, L.A. del Pozo ${ }^{5}$, H. Deng ${ }^{17}$, A. Dieckmann ${ }^{11}$, M. Dittmar ${ }^{4}$, M.S. Dixit ${ }^{7}$, E. do Couto e Silva ${ }^{12}$, J.E. Duboscq ${ }^{8}$, E. Duchovni' ${ }^{26}$, G. Duckeck ${ }^{11}$, I.P. Duerdoth ${ }^{16}$, D.J.P. Dumas ${ }^{6}$, P.A. Elcombe ${ }^{5}$, P.G. Estabrooks ${ }^{6}$, E. Etzion ${ }^{23}$, H.G. Evans ${ }^{9}$, F. Fabbri ${ }^{2}$, B. Fabbro ${ }^{21}$, M. Fierro ${ }^{2}$, M. Fincke-Keeler ${ }^{28}$, H.M. Fischer ${ }^{3}$, D.G. Fong ${ }^{17}$, M. Foucher ${ }^{17}$, A. Gaidot ${ }^{21}$, J.W. Gary ${ }^{4}$, J. Gascon ${ }^{18}$, N.I. Geddes ${ }^{20}$, C. Geich-Gimbel ${ }^{3}$, S.W. Gensler ${ }^{9}$, F.X. Gentit ${ }^{21}$, G. Giacomelli ${ }^{2}$, R. Giacomelli ${ }^{2}$, V. Gibson ${ }^{5}$, W.R. Gibson ${ }^{13}$, J.D. Gillies ${ }^{20}$, J. Goldberg ${ }^{22}$, D.M. Gingrich ${ }^{30, \text { a }}$, M.J. Goodrick, W. Gorn ${ }^{4}$, C. Grandi ${ }^{2}$, F.C. Grant ${ }^{5}$, J. Hagemann ${ }^{27}$, G.G. Hanson ${ }^{12}$, M. Hansroul ${ }^{8}$, C.K. Hargrove ${ }^{7}$, P.F. Harrison ${ }^{13}$, J. Hart ${ }^{8}$, P.M. Hattersley ${ }^{1}$, M. Hauschild ${ }^{8}$, C.M. Hawkes ${ }^{8}$, E. Heflin ${ }^{4}$, R.J. Hemingway 6 , G. Herten ${ }^{10}$, R.D. Heuer ${ }^{8}$, J.C. Hill ${ }^{5}$, S.J. Hillier ${ }^{8}$, T. Hilse ${ }^{10}$, D.A. Hinshaw ${ }^{18}$, J.D. Hobbs ${ }^{8}$, P.R. Hobson ${ }^{25}$, D. Hochman ${ }^{26}$, R.J. Homer ${ }^{1}$, A.K. Honma ${ }^{28, a}$, R.E. Hughes-Jones ${ }^{16}$, R. Humbert ${ }^{10}$, P. Igo-Kemenes ${ }^{11}$, H. Ihssen ${ }^{11}$, D.C. Imrie ${ }^{25}$, A.C. Janissen ${ }^{6}$, A. Jawahery ${ }^{17}$, P.W. Jeffreys ${ }^{20}$, H. Jeremie ${ }^{18}$, M. Jimack ${ }^{1}$, M. Jones ${ }^{29}$, R.W.L. Jones ${ }^{8}$, P. Jovanovic ${ }^{1}$, C. Jui ${ }^{4}$, D. Karlen ${ }^{6}$, K. Kawagoe ${ }^{24}$, T. Kawamoto ${ }^{24}$, R.K. Keeler ${ }^{28}$, R.G. Kellogg ${ }^{17}$, B.W. Kennedy ${ }^{15}$, J. King ${ }^{13}$, S. Kluth ${ }^{5}$, T. Kobayashi ${ }^{24}$, D.S. Koetke ${ }^{8}$, T.P. Kokott ${ }^{3}$, S. Komamiya ${ }^{24}$, J.F. Kral ${ }^{8}$, R. Kowalewski J. von Krogh ${ }^{11}$, J. Kroll ${ }^{9}$, P. Kyberd ${ }^{13}$, G.D. Lafferty ${ }^{16}$, H. Lafoux ${ }^{21}$, R. Lahmann ${ }^{17}$, J. Lauber ${ }^{8}$, J.G. Layter ${ }^{4}$, P. Leblanc ${ }^{18}$, A.M. Lee ${ }^{31}$, E. Lefebvre ${ }^{18}$, M.H. Lehto ${ }^{15}$, D. Lellouch ${ }^{26}$, C. Leroy ${ }^{18}$, J. Letts ${ }^{4}$, L. Levinson ${ }^{26}$, S.L. Lloyd ${ }^{13}$, F.K. Loebinger ${ }^{16}$, J.M. Lorah ${ }^{17}$, B. Lorazo ${ }^{18}$, M.J. Losty ${ }^{7}$, X.C. Lou' ${ }^{12}$, J. Ludwig ${ }^{10}$, A. Luig ${ }^{10}$, M. Mannelli $i^{8}$, S. Marcellini' ${ }^{2}$, C. Markus ${ }^{3}$, A.J. Martin ${ }^{13}$, J.P. Martin ${ }^{18}$, T. Mashimo ${ }^{24}$, P. Mättig', U. Maur ${ }^{3}$, J. McKenna ${ }^{29}$, T.J. McMahon', J.R. McNutt ${ }^{25}$, F. Meijers ${ }^{8}$, D. Menszner ${ }^{11}$, F.S. Merritt ${ }^{9}$, H. Mes ${ }^{7}$, A. Michelini ${ }^{8}$, R.P. Middleton ${ }^{20}$, G. Mikenberg ${ }^{26}$, J. Mildenberger ${ }^{6}$, D.J. Miller ${ }^{15}$, R. Mir ${ }^{2}$, W. Mohr ${ }^{10}$, C. Moisan ${ }^{18}$,

A. Montanari ${ }^{2}$, T. Mori ${ }^{24}$, M. Mori' ${ }^{24}$, U. Müller ${ }^{3}$, B. Nellen ${ }^{3}$, H.H. Nguyen ${ }^{9}$, S.W. O'Neale ${ }^{1}$, F.G. Oakham ${ }^{7}$, F. Odorici ${ }^{2}$, H.O. Ogren ${ }^{12}$, C.J. Oram ${ }^{28, a}$, M.J. Oreglia', S. Orito ${ }^{24}$, J.P. Pansart ${ }^{21}$, B. Panzer-Steindel ${ }^{8}$, P. Paschievici ${ }^{26}$, G.N. Patrick ${ }^{20}$, N. Paz-Jaoshvili ${ }^{23}$, M.J. Pearce ${ }^{1}$, P. Pfister ${ }^{10}$, J.E. Pilcher ${ }^{9}$, J. Pinfold ${ }^{30}$, D. Pitman ${ }^{28}$, D.E. Plane ${ }^{8}$, P. Poffenberger ${ }^{28}$, B. Poli ${ }^{2}$, T.W. Pritchard ${ }^{13}$, H. Przysiezniak ${ }^{18}$, G. Quast ${ }^{27}$, M.W. Redmond ${ }^{8}$, D.L. Rees ${ }^{8}$, G.E. Richards ${ }^{16}$, M. Rison ${ }^{5}$, S.A. Robins ${ }^{5}$, D. Robinson ${ }^{8}$, A. Rollnik ${ }^{3}$, J.M. Roney ${ }^{28}$, E. Ros ${ }^{8}$, S. Rossberg ${ }^{10}$, A.M. Rossi ${ }^{2}$, M. Rosvick ${ }^{28}$, P. Routenburg ${ }^{30}$, K. Runge ${ }^{10}$, O. Runolfsson ${ }^{8}$, D.R. Rust ${ }^{12}$, M. Sasaki ${ }^{24}$, C. Sbarra ${ }^{2}$, A.D. Schaile ${ }^{26}$, O. Schaile ${ }^{10}$, W. Schappert ${ }^{6}$, F. Scharf ${ }^{3}$, P. Scharff-Hansen ${ }^{8}$, P. Schenk ${ }^{4}$, B. Schmitt ${ }^{3}$, H. von der Schmitt ${ }^{11}$, M. Schröder ${ }^{12}$, C. Schwick ${ }^{27}$, J. Schwiening ${ }^{3}$, W.G. Scott ${ }^{20}$, M. Settles ${ }^{12}$, T.G. Shears ${ }^{5}$, B.C. Shen ${ }^{4}$, C.H. Shepherd-Themistocleous ${ }^{7}$, P. Sherwood ${ }^{15}$, G.P. Siroli ${ }^{2}$, A. Skillman ${ }^{16}$, A. Skuja ${ }^{17}$, A.M. Smith ${ }^{8}$, T.J. Smith ${ }^{28}$, G.A. Snow ${ }^{17}$, R. Sobie ${ }^{28}$, R.W. Springer ${ }^{17}$, M. Sproston ${ }^{20}$, A. Stahl ${ }^{3}$, C. Stegmann ${ }^{10}$, K. Stephens ${ }^{16}$, J. Steuerer $^{28}$, R. Ströhmer ${ }^{11}$, D. Strom ${ }^{19}$, H. Takeda ${ }^{24}$, T. Takeshita ${ }^{24, c}$, S. Tarem ${ }^{26}$, M. Tecchio ${ }^{9}$, P. Teixeira-Dias ${ }^{11}$, N. Tesch ${ }^{3}$, M.A. Thomson ${ }^{15}$, E. Torrente-Lujan ${ }^{22}$, S. Towers ${ }^{28}$, G. Transtromer ${ }^{25}$, N.J. Tresilian ${ }^{16}$, T. Tsukamoto ${ }^{24}$, M.F. Turner ${ }^{8}$, D. Van den plas $^{18}$, R. Van Kooten ${ }^{27}$, G.J. VanDalen ${ }^{4}$, G. Vasseur ${ }^{21}$, A. Wagner ${ }^{27}$, D.L. Wagner ${ }^{9}$,

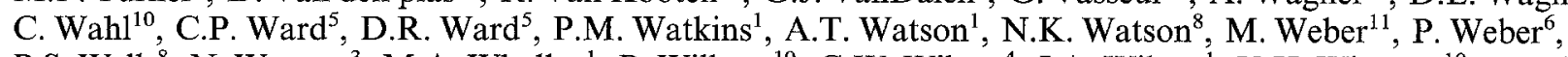
P.S. Wells ${ }^{8}$, N. Wermes ${ }^{3}$, M.A. Whalley ${ }^{1}$, B. Wilkens ${ }^{10}$, G.W. Wilson ${ }^{4}$, J.A. Wilson ${ }^{1}$, V-H. Winterer ${ }^{10}$, T. Wlodek ${ }^{26}$, G. Wolf ${ }^{26}$, S. Wotton ${ }^{11}$, T.R. Wyatt ${ }^{16}$, R. Yaari ${ }^{26}$, A. Yeaman ${ }^{13}$, G. Yekutieli ${ }^{26}$, M. Yurko ${ }^{18}$, W. Zeuner ${ }^{8}$, G.T. Zorn ${ }^{17}$

\footnotetext{
${ }^{1}$ School of Physics and Space Research, University of Birmingham, Birmingham, B 15 2TT, UK

${ }^{2}$ Dipartimento di Fisica dell' Università di Bologna and INFN, I-40126 Bologna, Italy

${ }^{3}$ Physikalisches Institut, Universität Bonn, D-53115 Bonn 1, Germany

${ }^{4}$ Department of Physics, University of California, Riverside, CA92521 USA

${ }^{5}$ Cavendish Laboratory, Cambridge, CB3 0HE, UK

${ }^{6}$ Carleton University, Department of Physics, Colonel By Drive, Ottawa, Ontario K1S 5B6, Canada

${ }^{7}$ Centre for Research in Particle Physics, Carleton University, Ottawa, Ontario K 1S 5B6, Canada

${ }^{8} \mathrm{CERN}$, European Organisation for Particle Physics, CH-1211 Geneva 23, Switzerland
} 
${ }^{9}$ Enrico Fermi Institute and Department of Physics, University of Chicago, Chicago IL60637, USA

${ }^{10}$ Fakultät für Physik, Albert Ludwigs Universität, D-79104 Freiburg, Germany

${ }^{11}$ Physikalisches Institut, Universität Heidelberg, D-69120 Heidelberg, Germany

${ }^{12}$ Indiana University, Department of Physics, Swain Hall West 117, Bloomington, IN 47405, USA

${ }^{13}$ Queen Mary and Westfield College, University of London, London, E1 4NS, UK

${ }^{14}$ Birkbeck College, London, WC1E 7HV, UK

${ }^{15}$ University College London, London, WC1E 6BT, UK

${ }^{16}$ Department of Physics, Schuster Laboratory, The University, Manchester, M13 9PL, UK

${ }^{17}$ Department of Physics, University of Maryland, College Park, MD 20742, USA

${ }^{18}$ Laboratoire de Physique Nucléaire, Université de Montréal, Montréal, Quebec, H3C 3J7, Canada

${ }^{19}$ University of Oregon, Department of Physics, Eugene, OR 97403, USA

${ }^{20}$ Rutherford Appleton Laboratory, Chilton, Didcot, Oxfordshire, OX 110 QX, UK

${ }^{21}$ DAPNIA/SPP, Saclay, F-91191 Gif-sur-Yvette, France

${ }_{22}$ Department of Physics, Technion-Israel Institute of Technology, Haifa 32000, Israel

${ }^{23}$ Department of Physics and Astronomy, Tel Aviv University, Tel Aviv 69978, Israel

${ }^{24}$ International Centre of Elementary Particle Physics and Department of Physics, University of Tokyo, Tokyo 113, and Kobe

University, Kobe 657, Japan

${ }^{25}$ Brunel University, Uxbridge, Middlesex, UB8 3PH UK

${ }^{26}$ Nuclear Physics Department, Weizmann Institute of Science, Rehovot, 76100, Israel

${ }^{27}$ Universität Hamburg/DESY, II Institut für Experimental Physik, D-22607 Hamburg, Germany

${ }^{28}$ University of Victoria, Department of Physics, PO Box 3055, Victoria BC V8W 3P6, Canada

${ }^{29}$ University of British Columbia, Department of Physics, Vancouver BC V6T 1Z1, Canada

${ }^{30}$ University of Alberta, Department of Physics, Edmonton AB T6G 2N5, Canada

${ }^{31}$ Duke University, Department of Physics, Durham, NC 27708-0305, USA

Received 26 July 1993

\begin{abstract}
We have studied the reactions $\gamma \gamma \rightarrow \mu^{+} \mu^{-}$and $\gamma \gamma \rightarrow \tau^{+} \tau^{-}$by measuring the properties of events of the types $e^{+} e^{-} \rightarrow e^{+} e^{-} \mu^{+} \mu^{-}$and $e^{+} e^{-} \rightarrow e^{+} e^{-} \tau^{+} \tau^{-}$at $e^{+} e^{-}$centre-of-mass energies between 88 and $94 \mathrm{GeV}$. The data sample corresponds to an integrated $e^{+} e^{-}$luminosity of $40.4 \mathrm{pb}^{-1}$ collected by the OPAL detector at LEP. The QED structure function $F_{2}$ is extracted from a sample of $1462 \gamma \gamma \rightarrow \mu^{+} \mu^{-}$events in which one photon is off the mass shell (single-tagged). The $Q^{2}$ range for these massive photons is $4-400 \mathrm{GeV}^{2}$, with an average $Q^{2}$ of $8 \mathrm{GeV}^{2}$. We have observed $48 e^{+} e^{-} \rightarrow e^{+} e^{-} \mu^{+} \mu^{-}$ events in which both final state electrons are detected. In the sample of single-tag events we have identified $34.9 \pm 6.7$ events as due to the reaction $\gamma \gamma \rightarrow \tau^{+} \tau^{-}$, on an estimated background of $5.1 \pm 2.7$ events. In all cases the measured event distributions agree with QED calculations.
\end{abstract}

\section{Introduction}

Two-photon reactions have been studied at $e^{+} e^{-}$colliders for some time [1,2] and measurements of the reaction $\gamma \gamma \rightarrow \mu^{+} \mu^{-}$have been made at $e^{+} e^{-}$centre-of-mass energies of up to $61.4 \mathrm{GeV}$ [3-10]. The two-photon reactions $e^{+} e^{-} \rightarrow e^{+} e^{-} \mu^{+} \mu^{-}$and $e^{+} e^{-} \rightarrow e^{+} e^{-} \tau^{+} \tau^{-}$are pure $\mathscr{O}\left(\alpha^{4}\right)$ QED reactions and are, therefore, in principle well understood.

In this paper, we present a measurement of the reaction $e^{+} e^{-} \rightarrow e^{+} e^{-} \mu^{+} \mu^{-}$under two conditions: $i$ ) single

\footnotetext{
a Also at TRIUMF, Vancouver, Canada V6T $2 \mathrm{~A} 3$

b Now at MPI, München, Germany

c Also at Shinshu University, Matsumoto 390, Japan
}

tag, i.e. one of the final state electrons was observed, and ii) double tag, i.e. both final state electrons were observed. In both cases, we required the two final state muons to be observed. Untagged events, those in which both final state electrons escaped detection close to the beam axis were not considered.

This paper also describes the first direct observation of tau pair production in photon-photon collisions. The high energy of the LEP beams makes the $\gamma \gamma$ mass range needed for $\tau^{+} \tau^{-}$production more accessible. This feature of LEP and the excellent particle identification properties of the OPAL detector enable us to measure the reaction $e^{+} e^{-} \rightarrow e^{+} e^{-} \tau^{+} \tau^{-}$by selecting final states with one electron and one muon or charged hadron, in addition to the tag. In order to reduce backgrounds only single-tag events were considered in this study.

The data sample consists of the data collected from 1990 to 1992 , which amounts to $40.4 \mathrm{pb}^{-1}$ of integrated $e^{+} e^{-}$luminosity in the centre-of-mass energy range between 88 and $93 \mathrm{GeV}$.

\section{Theory and Monte Carlo simulations}

The four main diagrams contributing at the lowest order to the reactions $e^{+} e^{-} \rightarrow e^{+} e^{-} \mu^{+} \mu^{-}$and $e^{+} e^{-} \rightarrow$ $e^{+} e^{-} \tau^{+} \tau^{-}$are shown in Fig. 1. The classical "two-photon" diagram is the one shown as "multiperipheral", Fig. 1a. We compare the data to the following Monte Carlo simulations:

1. The single-tag $e^{+} e^{-} \rightarrow e^{+} e^{-} \mu^{+} \mu^{-}$events were simulated using the four-fermion generator written by Berends, Daverveldt and Kleiss [11] (BDK), which includes all four diagrams. We have used the version modified by Daverveldt to include the contributions coming from the annihilation through the $Z^{0}$. It is optimised for the gen- 


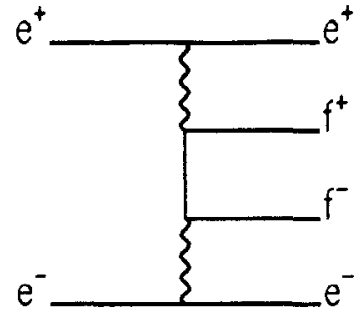

a) Multiperipheral

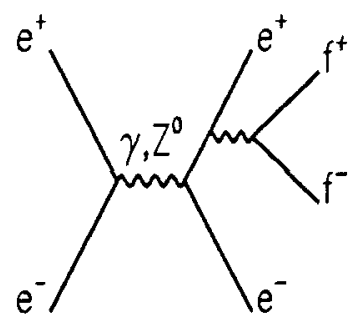

c) Annihilation

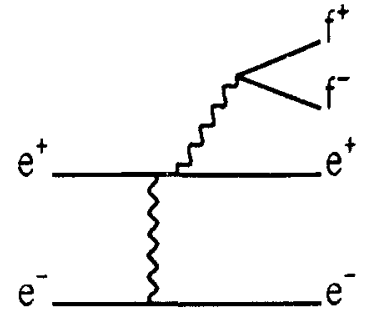

b) Bremsstrahlung

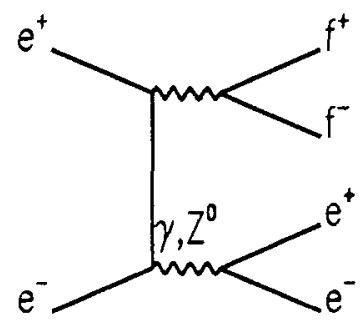

d) Conversion
Fig.1a-d. The four main diagrams contributing at the lowest order to the processes $e^{+} e^{-} \rightarrow e^{+} e^{-} f^{+} f^{-}$, with $f=\mu, \tau$. In $b$ both initial and final state radiation are included

eration of untagged events and usable for single-tag events, but it is very inefficient in generating double-tag events.

2. For the simulation of double-tag data, we used the FERMISV [12] generator, which includes the same diagrams, and also initial and final state radiation.

3. The $e^{+} e^{-} \rightarrow e^{+} e^{-} \tau^{+} \tau^{-}$events were simulated using the Vermaseren generator [13] which includes the diagrams of Fig. 1a and b. The decay of the tau leptons was simulated with the routines from the JETSET library $[14,15]$.

The events from all three generators were processed by the OPAL detector simulation code [16] and the standard offline reconstruction code. All events were generated at a centre-of-mass energy corresponding to the $Z^{0}$ mass, where 33.1 of the $40.4 \mathrm{pb}^{-1}$ of data were taken. The BDK generator was used to verify that the bremsstrablung diagram contributes $2 \%$ of the events to the single-tag $e^{+} e^{-} \rightarrow e^{+} e^{-} \mu^{+} \mu^{-}$channel. The annhihilation and conversion diagrams both contribute less than $0.1 \%$, and were neglected in the single-tag analysis.

For comparing the photon structure function $F_{2}$ obtained from the data with the QED prediction, we have used TWOGEN, a Monte Carlo which generates only the two-photon flux factor $N$ which can then be folded with any structure function $[17,18]$. The differential cross section for single-tag events can be written as:

$$
\begin{aligned}
\frac{\mathrm{d}^{3} \sigma}{\mathrm{d} x \mathrm{~d} Q^{2} \mathrm{~d} z}= & \frac{4 \pi \alpha^{2}}{Q^{4} x}\left\{(1-y) F_{2}\left(x, Q^{2}\right)\right. \\
& \left.+x y^{2} F_{1}\left(x, Q^{2}\right)\right\} \frac{\mathrm{d} N}{\mathrm{~d} z} .
\end{aligned}
$$

Here $Q^{2}$ is the momentum transfer squared of the photon, tagged by the measurement of the scattered electron. The kinematic variable $x$ is defined as $x=Q^{2} /\left(Q^{2}+W^{2}\right)$, where $W$ is the invariant mass of muon pair, $y \approx 1$ $-E_{\text {tag }} / E_{\text {beam }}$ and $z=1-E_{\text {untag }} / E_{\text {beam }}$. The energies of the tagged and the untagged electron are $E_{\mathrm{tag}}$ and $E_{\text {untag }}$, respectively. The untagged electron is assumed to have $Q^{2}=0$. The QED structure functions of the photon are predicted to be [19]:

$$
\begin{aligned}
& F_{1}\left(x, Q^{2}\right) \\
& =\frac{\alpha}{2 \pi}\left\{\left[x^{2}+(1-x)^{2}+4 m_{\mu}^{2} \frac{W^{2}-2 m_{\mu}^{2}}{\left(W^{2}+Q^{2}\right)^{2}}\right]\right. \\
& \quad \times \log \left[\frac{W}{2 m_{\mu}}+\left(\frac{W^{2}}{4 m_{\mu}^{2}}-1\right)^{1 / 2}\right]^{2} \\
& \left.\quad-\left[(1-2 x)^{2}+\frac{4 m_{\mu}^{2} W^{2}}{\left(W^{2}+Q^{2}\right)^{2}}\right]\left(1-\frac{4 m_{\mu}^{2}}{W^{2}}\right)^{1 / 2}\right\},
\end{aligned}
$$

and

$$
\begin{aligned}
& F_{2}\left(x, Q^{2}\right) \\
& =2 x F_{1}\left(x, Q^{2}\right)+\frac{4 \alpha}{\pi} x^{2}\left\{(1-x)\left(1-\frac{4 m_{\mu}^{2}}{W^{2}}\right)^{1 / 2}\right. \\
& \left.-\frac{2 m_{\mu}^{2}}{W^{2}+Q^{2}} \log \left[\frac{W}{2 m_{\mu}}+\left(\frac{W^{2}}{4 m_{\mu}^{2}}-1\right)^{1 / 2}\right]^{2}\right\} .
\end{aligned}
$$

The contribution from the structure function $F_{1}\left(x, Q^{2}\right)$ is negligible for $y<0.25$. In this paper, we shall only be concerned with the determination of $F_{2}\left(x, Q^{2}\right)$.

\section{The OPAL detector}

As the OPAL detector has already been described in detail [20], we restrict ourselves here to an overview of the main components. The coordinate system of the detector is defined with the $z$-axis along the electron beam direction, $\vartheta$ and $\varphi$ being the polar and azimuthal angles. Tracking of charged particles is mainly performed by the jet chamber, a large volume drift chamber, $4 \mathrm{~m}$ long and $3.7 \mathrm{~m}$ in diameter, divided into 24 azimuthal sectors each containing a plane of 159 sense wires. The charge measured by the sense wires is used to obtain $\mathrm{d} E / \mathrm{d} x$, the specific energy loss of the particle in the detector gas. Together with the momentum, this gives a measurement of the mass of the particle, leading to an $e-\pi$ separating power of more than two standard deviations for momenta up to $12 \mathrm{GeV}$ [21]. The jet chamber, the vertex detector, and the chambers measuring the $z$ coordinates of particles as they leave the jet chamber, are positioned inside a solenoidal coil which provides an axial magnetic field of $0.435 \mathrm{~T}$. It is surrounded by a time-of-flight counter array (TOF), a presampler and an electromagnetic calorimeter.

The electromagnetic calorimeter consists of a barrel and two endcap arrays of lead glass blocks. Each block subtends a solid angle of approximately $40 \times 40 \mathrm{mrad}^{2}$, with a thickness of over 24 radiation lengths $\left(X_{0}\right)$ in the barrel region and typically $22 X_{0}$ in the endcaps. The energy resolution for electrons of $45 \mathrm{GeV}$ is typically 
$\sigma_{E} / E \approx 3 \%$. Outside the electromagnetic calorimeter is the instrumented return yoke of the magnet, forming the hadron calorimeter, and beyond this are four layers of the outer muon detectors. The hadron calorimater, which consists of nine layers (eight layers in the endcap) of streamer tubes interleaved with the iron slabs of the magnet return yoke, is read out via $4 \mathrm{~mm}$ wide strips spaced $1 \mathrm{~cm}$ apart, and projective towers formed by pads. These strips provide tracking in the $(r, \varphi)$ plane, while the towers measure the energy deposited in coarse $(\varphi, z)$ bins. The material in front of the hadron calorimeter corresponds to about two hadronic interaction lengths. The hadron calorimeter itself accounts for about 4.8 interaction lengths. A muon with a transverse momentum $p_{t}=1 \mathrm{GeV}$ will on the average traverse six planes of the hadron calorimeter.

The forward detectors consist of several sub-detectors of which three are used in this analysis: a lead-scintillator sandwich calorimeter, a set of proportional tube chambers embedded in the calorimeter, and two planes of drift chambers upstream of the calorimeter.

The forward detector calorimeter measures the energy deposited by a photon or an electron with a resolution of $\Delta E / E=18 \% / \sqrt{E}, E$ in $\mathrm{GeV}$. Careful calibration of the energy scale keeps the fluctuations in the measured shower energy between LEP fills to less than $0.5 \%$. The calorimeter also measures the shower position by virtue of its azimuthal division into 16 segments and separate inner and outer edge readouts. The polar angle resolution varies between 1 and $10 \mathrm{mrad}$, being best near the inner edge of the calorimeter. The resolution in azimuth varies between 3.5 and $35 \mathrm{mrad}$, being best at the segment boundaries. Furthermore, the calorimeter gives shower development information through longitudinal segmentation into presampler $\left(4 X_{0}\right)$ and main $\left(19 X_{0}\right)$ sections. The acceptance of the calorimeter extends from 39 to $155 \mathrm{mrad}$, and is essentially complete in azimuth. The tube chambers are situated after the presampler section and measure the shower position in $\vartheta$ and $\varphi$. The resolution for $45 \mathrm{GeV}$ electrons is $1.3 \mathrm{mrad}$ in $\vartheta$ and $19 \mathrm{mrad}$ in $\varphi$. The tube chamber acceptance extends from 50 to $135 \mathrm{mrad}$ in $\vartheta$ and covers $95 \%$ in azimuth. The precisely located drift chambers are used to determine the absolute radial position of the tube chambers to a precision of $135 \mu \mathrm{m}$. The distance between the forward detectors at each side of OPAL is known to a precision of $0.5 \mathrm{~mm}$.

\section{The event selection}

In the events under study we distinguish: i) the highenergy electrons (tags), and $i i)$ the low-energy muons of the decay products of the tau pair. These can be handled independently.

The pattern recognition program reconstructed charged tracks from strings of at least eight hits in the jet chamber $(\vartheta>200 \mathrm{mrad})$. A good track was defined in this analysis as having: at least 30 hits registered in the jet chamber, the first one at a radius smaller than $75 \mathrm{~cm}$; a distance of closest approach to the nominal beam axis of less than $1 \mathrm{~cm}$, and $|z|<20 \mathrm{~cm}$ at this point. The momentum of the track had to be between $300 \mathrm{MeV}$ and $20 \mathrm{GeV}$ with a component transverse to the beam axis $\left(p_{t}\right)$ greater than $100 \mathrm{MeV}$.

If a track failed one or more of these criteria, it could be defined as being of medium quality if the radius of its first measured point was at a distance of less than $50 \mathrm{~cm}$ from the beam axis and it had at least 30 jet chamber hits associated with it. As a result, medium quality tracks would include low energy photon conversion tracks and badly measured low angle tracks, but not tracks which scattered back from the pressure vessel or magnet coil. The latter, and other tracks not classified as of medium or good quality were ignored.

An electromagnetic calorimeter cluster was defined as an energy deposition of more than $100 \mathrm{MeV}$ in the barrel lead glass array, or more than $250 \mathrm{MeV}$ in the endcap.

In the first stage of the selection the presence of a tag was established. A tag in the forward detector was defined as any electromagnetic energy deposition $E_{\text {tag }}$ exceeding half the beam energy $E_{\text {beam }}$. We expect most tags to be observed in the forward detector because the tag angle spectrum is strongly peaked at small angles. In order to have well measured energy values, we required the polar angle $\vartheta_{\text {tag }}$ of this tag to be between 47 and $120 \mathrm{mrad}$. No distinction between photons and electrons was made in the forward detector. In order to ensure that the untagged electron in single-tag events had $Q^{2} \approx 0$, we rejected events with additional clusters in the forward detector with energies of more than the threshold value of $2 \mathrm{GeV}$ but less than $E_{\text {beam }} / 2$.

A tag with a polar angle above $200 \mathrm{mrad}$ was defined using electromagnetic clusters in the barrel and endcap lead glass arrays. Such a cluster was called a tag if its energy exceeded half the beam energy and at least one charged track of any quality was found in a cone of $15^{\circ}$ half-opening angle centred on the cluster. Additional charged tracks within this cone were ignored. No requirement was made on the charge of the tag.

In the next step of the selection only tracks not assigned to tags were considered for event selection. The events were required to have two and only two good tracks with opposite charges. Events with additional medium quality tracks were rejected, as were events with reconstructed photon conversions. At least one of the tracks had to have a momentum greater than $1 \mathrm{GeV}$ and a polar angle $\vartheta$ satisfying $|\cos \vartheta|<0.72$. The other charged track had to have a momentum larger than $300 \mathrm{MeV}$ and a polar angle satisfying $|\cos \vartheta|<0.95$.

The reaction $e^{+} e^{-} \rightarrow \tau^{+} \tau^{-}$was found to be a background to events in which the tag was measured at $\vartheta>200 \mathrm{mrad}$. This background was rejected by the requirement that $\vartheta_{\text {untag }}<200 \mathrm{mrad}$. Here $\vartheta_{\text {untag }}$ is the polar angle of the untagged electron, reconstructed from the momentum vectors of the tagged electron and the two charged tracks. The value of $200 \mathrm{mrad}$ allows for uncertainties in the momentum measurements.

All events were required to have initiated the detector read-out with a trigger consisting of a coincidence between an energy deposition of more than $15 \mathrm{GeV}$ in one of the two forward detector modules and a charged track in the barrel region of the jet chamber. The efficiency 
of this trigger for events satisfying all cuts was determined by means of other, independent, triggers to be $95.3 \pm 0.6 \%$.

\section{The event identification}

Identification of the final state particles was required for further selection. Identification criteria were defined for electrons and muons. Figures $2 \mathrm{a}$ and $\mathrm{b}$ show the overall efficiencies for identifying electrons and muons as functions of their momentum. The slight disagreement between data and Monte Carlo at high electron momenta has no influence on the results.

The electron identification was based on two measured quantities: the ratio of electromagnetic energy measured in the lead-glass calorimeter to the momentum measured in the central detector $E / p$, and the energy loss $\mathrm{d} E / \mathrm{d} x$ in the jet chamber. The $E / p$ selection was used for momenta greater than $3 \mathrm{GeV}$ : a charged particle was called an electron if the electromagnetic cluster associated with it had an energy $E$ satisfying $E / p>0.8$. Between 0.3 and $3.0 \mathrm{GeV}$ a particle was identified as an electron if $9.0<\mathrm{d} E / \mathrm{d} x<12.0 \mathrm{keV} / \mathrm{cm}$. For both momentum ranges, it was required that no signal above $200 \mathrm{MeV}$ was observed in the region of the hadron calorimeter associated with the charged track.

Charged particles were identified as muons only if their momentum exceeded $1 \mathrm{GeV}$. The electromagnetic energy associated to the charged track was required to be less than $1.5 \mathrm{GeV}$. A signal was required in the muon chambers or in the hadron calorimeter strips. In the muon chambers, a track segment with at least three hits had to be reconstructed. In the hadron calorimeter, at least three layers had to show a strip signal associated with the track, of which one had to be at least in the fourth layer. The ratio of the total number of strips to the number of layers hit had to be less than three. This last requirement was to reject hadrons which penetrated several layers before initiating a shower. In order to satisfy the criteria for muon identification, a muon impinging perpendicularly on the hadron calorimeter had to traverse the lead glass array and at least three layers of iron, amounting to a total of four hadronic interaction lengths.

\section{Single-tag mupair events}

We identified the $e^{+} e^{-} \rightarrow e^{+} e^{-} \mu^{+} \mu^{-}$final state by requiring the presence of one identified muon and rejecting events with identified electrons other than the tag. With these cuts we selected 1462 events, of which 48 events had a tag with $\vartheta_{\text {tag }}>200 \mathrm{mrad}$.

\subsection{Backgrounds and systematic errors}

The process $e^{+} e^{-} \rightarrow e^{+} e^{-} \tau^{+} \tau^{-}$constitutes the dominant background to $e^{+} e^{-} \rightarrow e^{+} e^{-} \mu^{+} \mu^{-}$. Using the Vermaseren Monte Carlo we have estimated the background for all $\tau$ decay channels to be $24.0 \pm 2.4$ events. Cross sections for continuum production and resonance formation in $\gamma \gamma \rightarrow \pi^{+} \pi^{-}$are much lower than the $\gamma \gamma \rightarrow \mu^{+} \mu^{-}$cross section and the pions are not expected to pass the muon selection criteria described above. The reaction $e^{+} e^{-} \rightarrow$ $\tau^{+} \tau^{-}$contributes $0.2 \pm 0.2$ events to the events with $\vartheta_{\text {tag }}>200 \mathrm{mrad}$.

The systematic errors in the reconstruction and selection efficiency were derived from the comparison between the data and the BDK Monte Carlo. We varied the cuts and compared the ratios of the numbers of events in the data and the Monte Carlo. We have estimated the errors due to the cuts in three intervals of $x$. Adding the errors in quadrature, we obtained systematic errors for the efficiency of $8.2 \%$ for $x<0.4,11.9 \%$ for $0.4<x<0.7$ and $8.2 \%$ for $x>0.7$. An error of $1 \%$ for the uncertainty in the luminosity measurement [22] was also included.

We have studied the effect of initial state radiation by adding to the TWOGEN Monte Carlo the possibility of generating one bremsstrahlung photon collinear with the beam axis. We found that this changed the acceptance with our cuts by less than $1 \%$ and we have therefore neglected the effect of initial state radiation in this paper.

Taking into account the systematic errors and the statistical error of the Monte Carlo sample, we expect $1418 \pm 142$ events from the BDK Monte Carlo, of which $46 \pm 7.4$ with a tag at $\vartheta>200 \mathrm{mrad}$. The average values of $x$ and $Q^{2}$ of the high- $Q^{2}$ events are 0.65 (RMS 0.64) and 127.8 (RMS 125.9), respectively, to be compared with 0.68 (RMS 0.67) and 140.4 (RMS 138.0) in the BDK Monte Carlo.
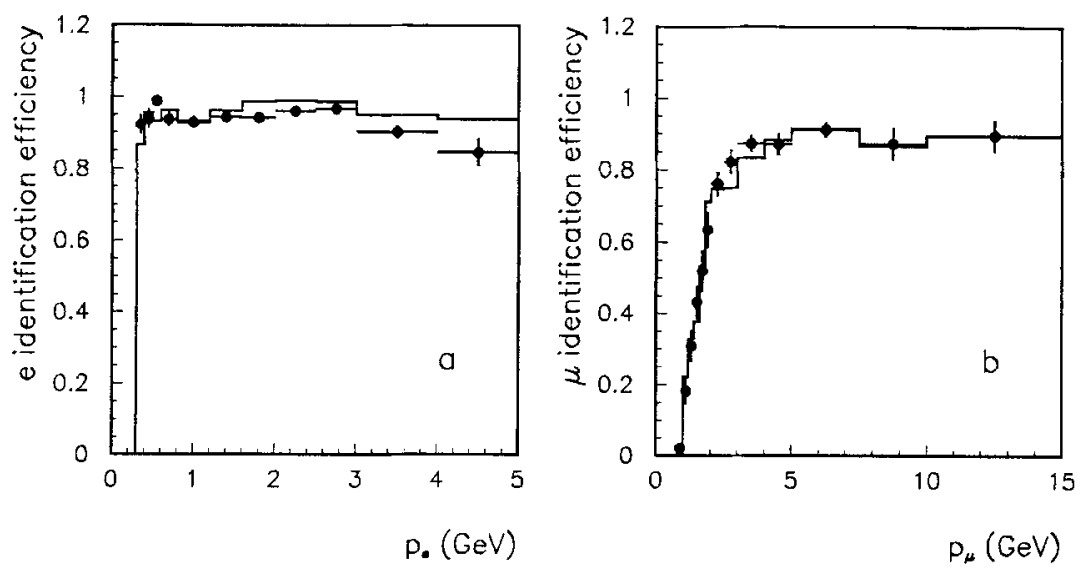

Fig. 2. a The efficiency for identifying an electron in a single-tag event in which the second particle was positively identified as an electron. $\mathbf{b}$ The efficiency for identifying a muon in a single-tag event in which the second particle was positively identified as a muon. The efficiencies are plotted as functions of the particle momenta. The points represent the data, the solid lines represent the Monte Carlo 


\subsection{The $Q E D$ structure function}

The QED structure function $F_{2}$ was extracted from the data using the following procedure:

1. Single-tag $e^{+} e^{-} \rightarrow e^{+} e^{-} \mu^{+} \mu^{-}$events were generated with the BDK Monte Carlo.

2. The generated events were subjected to the detector simulation and to the analysis cuts, and were weighted by a factor $\alpha / F_{2}\left(x, Q^{2}\right)$.

3. The $x$-distribution of the data was then divided by the Monte Carlo distribution of the weighted events. By doing this, we divided out the detector effects and also the photon flux factor, and obtained a distribution for $F_{2}$, which can be compared directly with QED calculations.

The result is shown in Fig. 3. The solid points are the measured values of $F_{2}$ as a function of $x$ for the tagging range $\vartheta_{\text {tag }}>47 \mathrm{mrad}$ and for $y<0.25$. This corresponds

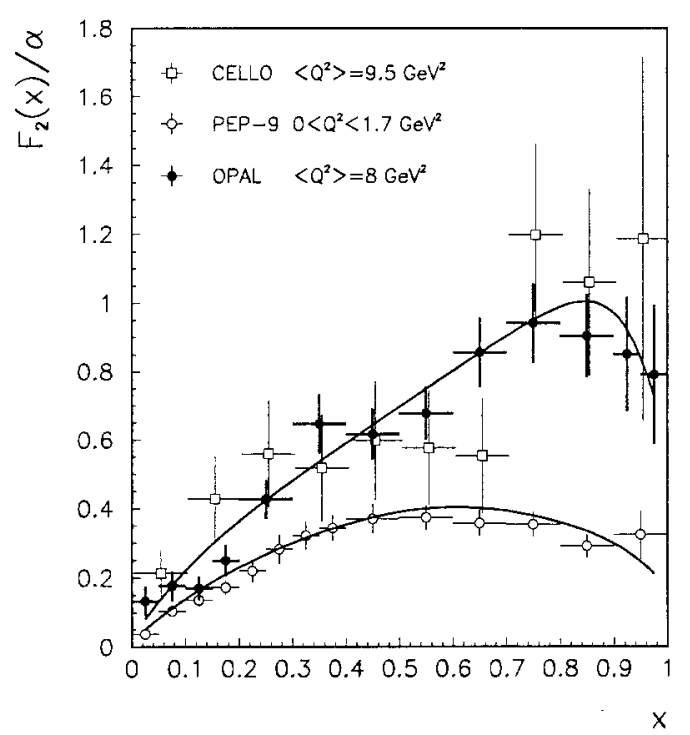

Fig. 3. The measured values of the structure functions $F_{2}$ as functions of $x$ for our measurement (full circles), the PEP-9 measurement (open circles) and the CELLO measurement (open squares). The solid lines are the QED expectations; the expectations for the CELLO and OPAL data are identical within the systematic errors to an average $Q^{2}$ (calculated from the generated events) of $8.0 \mathrm{GeV}^{2}$. The open circles are the PEP-9 measurements [4] for $0<Q^{2}<1.7 \mathrm{GeV}^{2}$. The open squares are the CELLO measurements [3] at $\left\langle Q^{2}\right\rangle=9.5 \mathrm{GeV}^{2}$. The solid lines represent the QED expectations. They were obtained by generating events according to the QED expression for $F_{2}$ with a two-photon luminosity generator as described in $[17,18]$. The expectation for the CELLO data is almost identical to the one shown for our data. The errors shown are statistical only. Our measurement is corrected for the estimated background from $\gamma \gamma \rightarrow \tau^{+} \tau^{-}$ events. It is in god agreement with the expectation.

\section{Double-tag mupair events}

We found 48 events in which both final state electrons were detected, either in the forward or in the central detector. We expect $36.9 \pm 5.0$ such events from the FERMISV Monte Carlo for the same integrated luminosity. In the kinematic region of the double-tag events the contributions of the annihilation and conversion diagrams become significant ( $9.3 \%$ and $2.8 \%$, respectively). This can be seen in the distribution of the events as a function of the acoplanarity of the tags, defined as $\pi$ $\varphi_{\operatorname{tag}_{1}}-\varphi_{\operatorname{tag}_{2}}$, shown in Fig. 4a. The distribution is uniform, except for very small acollinearities which have an excess of events. This behaviour is reproduced by the FERMISV Monte Carlo (open histogram), but not by the Vermaseren Monte Carlo, which does not include the annihilation and conversion diagrams. Figure $4 \mathrm{~b}$ shows the distribution of events as a function of the invariant $\mu^{+} \mu^{-}$mass. We see a slightly better agreement with the data in the FERMISV Monte Carlo than in the Vermaseren Monte Carlo, especially at the low masses. We attribute the slight excess of events in the mass bin between 0.5 and $1 \mathrm{GeV}$ to a statistical fluctuation. We do not observe the excess of events around $W=300 \mathrm{MeV}$ which was reported by the AMY collaboration [10].

As in the single-tag case, the reaction $e^{+} e^{-} \rightarrow$ $e^{+} e^{-} \tau^{+} \tau^{-}$is expected to be the dominant background to the double-tag $e^{+} e^{-} \rightarrow e^{+} e^{-} \mu^{+} \mu^{-}$events. Using the Vermaseren Monte Carlo we have estimated this background to be $1.7 \pm 0.6$ events. No Monte Carlo events of the reaction $e^{+} e^{-} \rightarrow \tau^{+} \tau^{-}$passed our cuts.
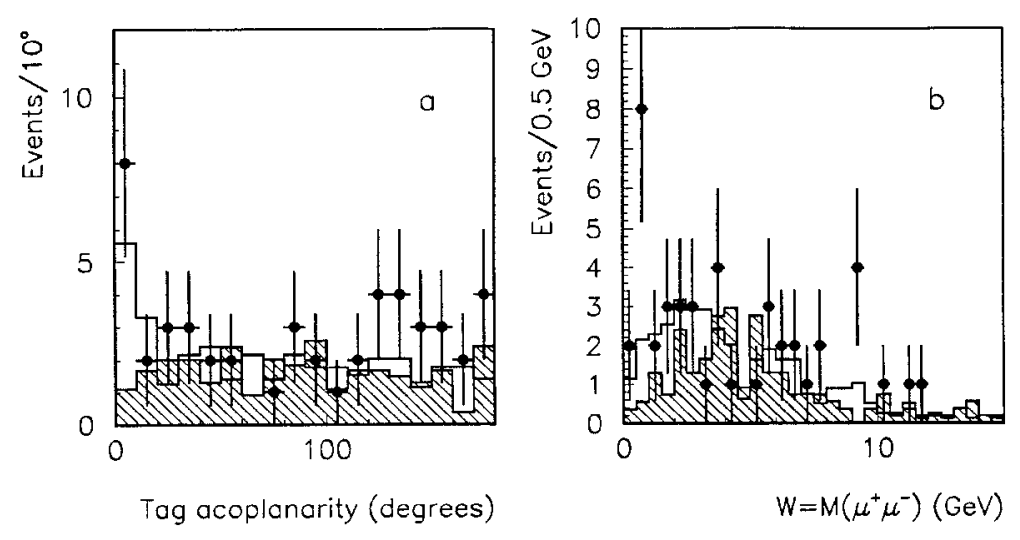

Fig. 4. a The distribution of double-tag $e^{+} e^{-} \rightarrow e^{+} e^{-} \mu^{+} \mu^{-}$events as a function of the acoplanarity between the two final state electrons. The points represent the accepted events, the lines the Monte Carlo simulations (open: FERMISV, shaded: Vermaseren), both normalised to the integrated luminosity. $\mathbf{b}$ The distribution of events as a function of $W$, the effective muon-muon mass. The data points and the histograms have the same meaning as in a. All errors are statistical only 


\section{The observation of $\gamma \gamma \rightarrow \tau^{+} \tau^{-}$events}

The selection criteria for identification of the $\tau^{+} \tau^{-}$final state were designed for events in which one tau decayed into an electron and the other tau into a muon or into a hadron with possibly additional neutral particles. This very characteristic decay mode represents about $25 \%$ of the total $\tau^{+} \tau^{-}$decays. For any other tau decay mode, the backgrounds from other two-photon reactions were too large for our cuts.

The final selection criterion is discernable in Fig. 5 which shows the distribution of tracks as a function of the particle identification variable $T_{R}$ in the case that the other track in the event was identified as an electron. Here, $T_{R} \equiv\left[T_{m}-T_{\mu}(p)\right] /\left[T_{e}(p)-T_{\mu}(p)\right], T_{m}$ is the measured energy loss rate in the jet chamber $(\mathrm{d} E / \mathrm{d} x)$, and $T_{e}(p)$ and $T_{\mu}(p)$ are the expected energy loss rates for an electron and a muon with momentum $p$, respectively. The points with error bars are the measured values; the shaded histogram corresponds to a Monte Carlo simulation of the $\gamma \gamma \rightarrow \tau^{+} \tau^{-}$process with the Vermaseren Monte Carlo, normalised to the integrated luminosity of the data sample and corrected for the triggering efficiency. The estimated contribution from the background was added to the Monte Carlo. The smooth lines represent fits of Gaussian distributions to the data. The resolution obtained from the fit is $3.5 \%$, in good agreement with the expectation [21]. The peak at about $T_{R}=0$ corresponds to the $\gamma \gamma \rightarrow \tau^{+} \tau^{-}$events accepted by our selection. The peak at $T_{R}=1$ in the Monte Carlo distribution is due to events in which both tau leptons decay to electrons. The cut was applied at $T_{R}=0.3$, which yields 43 events in the data.

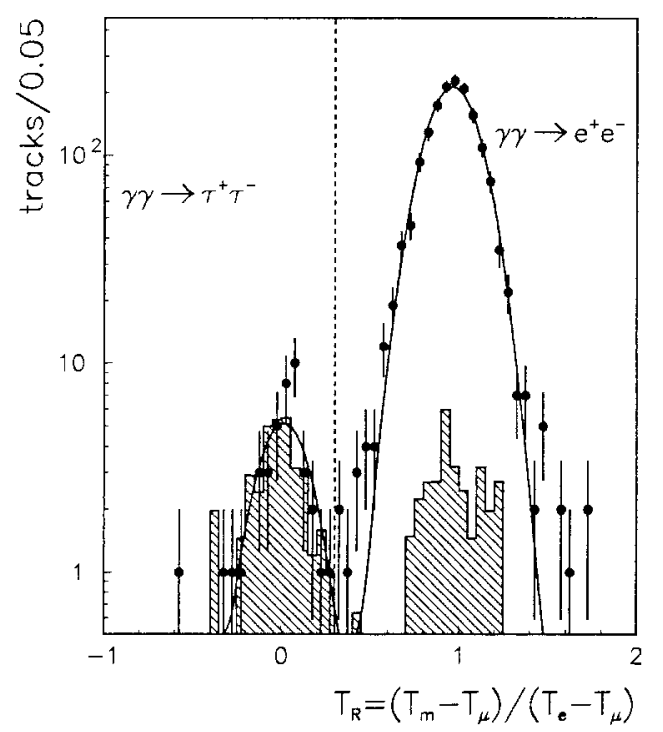

Fig. 5. The distribution of tracks as a function of $T_{R}$ in events in which the other track is identified as an electron. The points with error bars are the measured data, the shaded histogram corresponds to a Monte Carlo simulation of the $\gamma \gamma \rightarrow \tau^{+} \tau^{-}$process with the Vermaseren Monte Carlo, normalised to the integrated luminosity of the data sample. The estimated background is added to the Monte Carlo histogram. The smooth lines are fits of Gaussian distributions to the data. The dashed line indicates where the cut was applied

\subsection{Backgrounds for $e^{+} e^{-} \rightarrow e^{+} e^{-} \tau^{+} \tau^{-}$events}

We have considered several sources of backgrounds for the $e^{+} e^{-} \rightarrow e^{+} e^{-} \tau^{+} \tau^{-}$events:

1. The background due to the tail in the $T_{R}$ distribution of $\gamma \gamma \rightarrow e^{+} e^{-}$events was estimated by counting the number of tracks with $T_{R}>1.7$ in Fig. 5 . We found 2 events and expect statistically that the contamination of events with $T_{R}<0.3$ is the same. We found no $\gamma \gamma \rightarrow e^{+} e^{-}$Monte Carlo events satisfying all cuts.

2. The background due to the reaction $\gamma \gamma \rightarrow \mu^{+} \mu^{-}$was estimated from a Vermaseren Monte Carlo sample of $\gamma \gamma \rightarrow \mu^{+} \mu^{-}$events to be $1.9 \pm 1.1$ events.

3. The backgrounds from events with larger multiplicities with photon conversions in which one or more tracks were not reconstructed, was estimated by counting the number of events satisfying all cuts except the charge balance. We found one such event. This estimate includes the contribution from beam-gas interactions. In addition, the absence of beam-gas events is confirmed by the fact that the origin of the charged tracks (measured along the beam axis) corresponds within the experimental errors to the interaction point. In beam-gas events this origin is uniformly distributed along the beam axis.

4. The $e^{+} e^{-} \rightarrow \tau^{+} \tau^{-}(\gamma)$ Monte Carlo contributes $0.2 \pm 0.2$ event to the $\gamma \gamma \rightarrow \tau^{+} \tau^{-}$sample.

5. The reactions $\gamma \gamma \rightarrow K^{+} K^{-}$and $\gamma \gamma \rightarrow p \bar{p}$ can contribute to the sample if one of the kaons or protons lies in the $\mathrm{d} E / \mathrm{d} x$ overlap regions $e-K$ or $e-p$, and the other is identified as a muon or charged hadron. In two events, both particles were compatible with being kaons. The particles originally identified as electrons in these events had valid TOF signals which confirmed the kaon hypothesis. In the same way, one event was found to be compatible with $\gamma \gamma \rightarrow p \bar{p}$. We removed the events from the sample, and added the statistical error of \pm 1.7 to the error in the background.

Adding these numbers, we estimate a background of $5.1 \pm 2.7$ events in the $\gamma \gamma \rightarrow \tau^{+} \tau^{-}$event sample.

With the cuts described above we have selected 40 events in the data, with an estimated contamination of $5.1 \pm 2.7$ events due to various background reactions. We assume that the systematic error is of a similar magnitude as the systematic error established for the $\gamma \gamma \rightarrow \mu^{+} \mu^{-}$ acceptance. Including again a systematic error of $1 \%$ on the luminosity measurement for this sample we expect $32.7 \pm 4.8$ events from the Vermaseren Monte Carlo with the same cuts as applied to the data. The error also includes the statistical error of the Monte Carlo sample.

In Fig. 6a the distribution of the accepted $\gamma \gamma \rightarrow \tau^{+} \tau$ events is given as a function of the absolute value of the transverse momentum sum of the two charged particles and the tagged electron, normalised to the beam energy: $\left|\sum \mathbf{p}_{t}\right| / E_{\text {beam }}$. The points represent the data, the open histogram the Vermaseren Monte Carlo normalised to the integrated luminosity. The shaded histogram represents the same quantity for the single-tag $\gamma \gamma \rightarrow \mu^{+} \mu^{-}$ events, with an arbitrary normalisation. The striking difference between the $\gamma \gamma \rightarrow \mu^{+} \mu^{-}$and $\gamma \gamma \rightarrow \tau^{+} \tau^{-}$events can be explained by the presence of neutrinos and neutral 

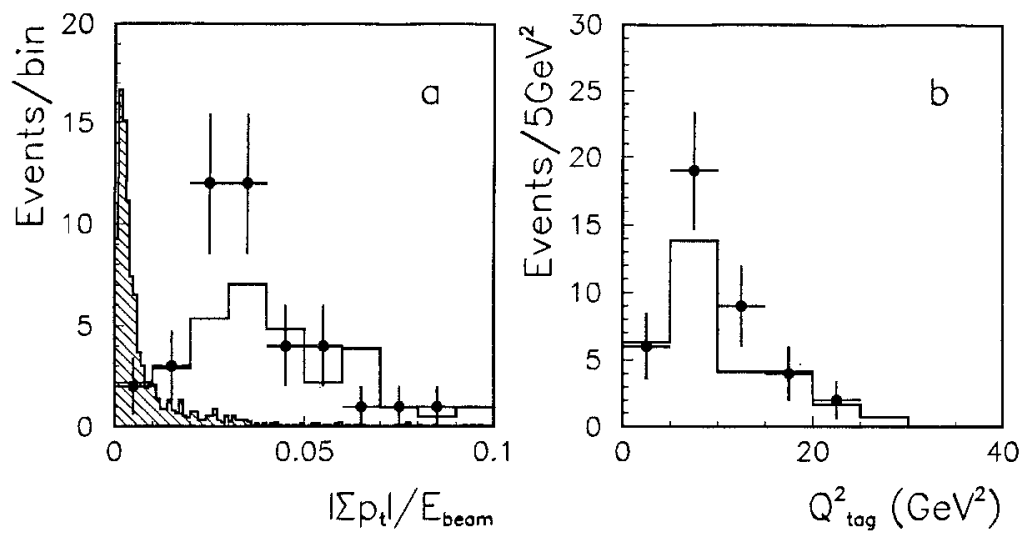

Fig. 6. a The distribution of $\gamma \gamma \rightarrow \tau^{+} \tau^{-}$events as a function of the scaled transverse momentum sum, including the tagged electron. The points represent the accepted $\gamma \gamma \rightarrow \tau^{+} \tau^{-}$events, the open histogram the $\gamma \gamma \rightarrow \tau^{+} \tau^{-}$Monte Carlo, normalised to the integrated luminosity. The shaded histogram shows the same quantity for $\gamma \gamma \rightarrow \mu^{+} \mu^{-}$events, with arbitrary normalisation. $\mathbf{b}$ The distribution of $\gamma \gamma \rightarrow \tau^{+} \tau^{-}$events as a function of $Q^{2}$ for the accepted events in the data (points with error bars) and in the Monte carlo (solid line). All errors are statistical only pions in the decays of the tau leptons, which are absent in the $\gamma \gamma \rightarrow \mu^{+} \mu^{-}$events. This difference and the fact that the $\gamma \gamma \rightarrow \tau^{+} \tau^{-}$distribution is well reproduced by the Monte Carlo confirm that the data are consistent with a significant production rate of $\gamma \gamma \rightarrow \tau^{+} \tau^{-}$events. In Fig. $6 \mathrm{~b}$ we show the distribution of the accepted $\gamma \gamma \rightarrow \tau^{+} \tau^{-}$events as a function of the $Q^{2}$ of the photon, tagged by the electron observed in the forward detector. The measured values (data points) are in reasonable agreement with the Monte Carlo expectation (solid line).

\section{Conclusions}

We have observed the reaction $e^{+} e^{-} \rightarrow e^{+} e^{-} \mu^{+} \mu^{-}$, both in the single-tag and in the double-tag mode. We have extracted the photon structure function $F_{2}$ from the single-tag events and found agreement with the QED expectation. We have selected 1462 single-tag events with an estimated background of $24.0 \pm 2.4$ events, and 48 double-tag events with an estimated background of $1.7 \pm 0.6$ events, which should be compared with the Monte Carlo expectations of $1418 \pm 142$ single-tag and $36.9 \pm 5.0$ double-tag events, respectively. We have identified $34.9 \pm 6.7$ events of the reaction $\gamma \gamma \rightarrow \tau^{+} \tau^{-}$in the single-tag mode on an estimated background of $5.1 \pm 2.7$ events. This is in good agreement with a QED Monte Carlo simulation using the Vermaseren generator, which predicts $32.7 \pm 4.8$ events. The $Q^{2}$ distribution of the $\gamma \gamma \rightarrow \tau^{+} \tau^{-}$events agrees with the expectation from the Vermaseren Monte Carlo.

Acknowledgements. It is a pleasure to thank the SL Division for the efficient operation of the LEP accelerator, the precise information on the absolute energy, and its continuing close cooperation with our experimental group. In addition to the support staff at our own institutions we are pleased to acknowledge the Department of Energy, USA, National Science Foundation, USA, Texas National Research Laboratory Commission, USA, Science and Engineering Research Council, UK, Natural Sciences and Engineering
Research Council, Canada, Fussefeld Foundation, Israeli Ministry of Energy and Ministry of Science, Minerva Gesellschaft, Japanese Ministry of Education, Science and Culture (the Monbusho) and a grant under the Monbusho International Science Research Program, German Israeli Bi-national Science Foundation (GIF), Direction des Sciences de la Matière du Commissariat à l'Energie Atomique, France, Bundesministerium für Forschung und Technologie, Germany, National Research Council of Canada, A.P. Sloan Foundation and Junta Nacional de Investigação Científica e Tecnológica, Portugal.

\section{References}

1. See for example: H. Kolanoski: Proc. of the IXth Int. Workshop on Photon-Photon Collisions, D.O.Caldwell, H.P. Paar (eds.), p. 3-34. UC San Diego, La Jolla, California, 1992

2. C. Berger, W. Wagner: Phys. Rep. 146 (1987) 1

3. CELLO Coll., H.-J. Behrend et al.: Phys. Lett. B126 (1983) 384

4. PEP-9 Coll., M.P. Cain et al.: Phys. Lett. B147 (1984) 232

5. PLUTO Coll., Ch. Berger et al.: Z.Phys. C27 (1985) 249

6. JADE Coll., W. Bartel et al.: Z.Phys. C30 (1986) 545

7. MARK-J Coll., B. Adeva et al.: Phys. Rev. D38 (1988) 2665

8. CELLO Coll., H.-J. Behrend et al.: Z.Phys. C43 (1989) 1

9. TOPAZ Coll., H. Hayashii et al.: Phys. Lett. B279 (1992) 422

10. AMY Coll., Y.H. Ho et al.: Phys. Lett. B244 (1990) 573

11. F.A. Berends, P.H. Daverveldt, R. Kleiss: Nucl. Phys. B253 (1985) 421

12. F. Le Diberder, J. Hilgart, R. Kleiss: CERN-PPE/92-115 (1992)

13. J.A.M. Vermaseren: Nucl. Phys. B229 (1983) 347

14. T. Sjöstrand: Comput. Phys. Commun. 39 (1986) 347

15. T. Sjöstrand, M. Bengtsson: Comput. Phys. Commun. 43 (1987) 367

16. J. Allison et al.: Nucl. Instrum. Methods A317 (1992) 47

17. A. Buijs et al.: TWOGEN, a Monte Carlo Generator for TwoPhoton Reactions (to be published)

18. W.G:J. Langeveld: Ph.D. Thesis (unpublished), Rijksuniversiteit Utrecht, 1985

19. V.M. Budnev, I.F. Ginzburg, G.V. Meledin, V.G. Serbo: Phys. Rep. 15 (1975) 181

20. OPAL Coll., K. Ahmet et al.: Nucl. Instrum. Methods A305 (1991) 275

21. M. Hauschild et al.: Nucl. Instrum. Methods A 314 (1992) 74

22. OPAL Coll., G. Alexander et al.: Z.Phys. C52 (1991) 175 